\title{
FORMAÇÃO PROFISSIONAL E INTEGRAÇÃO COM A REDE BÁSICA DE SAÚDE
}

\author{
PROFESSIONAL TRAINING AND INTEGRATION WITH THE BASIC HEALTH NETWORK
}

FORMACIÓN PROFESIONAL E INTEGRACIÓN CON LA RED BÁSICA DE SALUD

\author{
Leila Maria Vieira ${ }^{1}$ \\ Claudia de Almeida Prado e Piccino Sgavioli ${ }^{2}$ \\ Eliane Maria Ravasi Stefano Simionato ${ }^{3}$ \\ Elza Socorra Yamada Inoue ${ }^{4}$ \\ Maricê Thereza Correa Domingues Heubel ${ }^{5}$ \\ Marta Helena Souza De Conti ${ }^{6}$ \\ Sandra de Oliveira Saes ${ }^{7}$
}

Resumo A integração entre ensino e serviço proporciona melhor capacitação do docente, do estudante e do profissional do serviço de saúde, e assim garante ações e serviços de qualidade à população. Este estudo teve como objetivo reorientar a formação dos profissionais dos cursos do Centro de Ciências da Saúde da Universidade Sagrado Coração, Bauru, São Paulo, em consonância com o Programa Nacional de Reorientação da Formação Profissional em Saúde II. Nestes cursos, foram reorganizadas as suas matrizes curriculares, incluindo a adequação do ensino às demandas sociais, do mercado de trabalho e a incorporação de novas tecnologias educacionais. A partir desta análise, criou-se um grupo de disciplinas comum a todos os cursos de saúde da instituição, ou seja, desenvolveu-se uma identidade que caracteriza o perfil do profissional dos cursos do Centro de Ciências da Saúde da Universidade Sagrado Coração. Os resultados foram a formação do profissional com competência para atuar como gestor e multiplicador dos princípios do Sistema Único de Saúde, o incremento no processo de capacitação do profissional em serviço e a promoção do trabalho multiprofissional. Para os usuários, os resultados revertem-se em melhoria da qualidade dos serviços recebidos.

Palavras-chave matriz curricular; saúde; atenção básica.
Abstract Integration between teaching and service affords better training to teachers, students, and to health service professionals, and, as such, ensures quality actions and services are provided to the population. This study aimed to refocus the training of professionals taking courses at the Health Sciences Center at Sagrado Coração University, in Bauru, state of São Paulo, Brazil, in line with the National Reorientation Program for Vocational Training in Health II. These courses' curricula were reorganized, including adjustments made to teaching in accordance with social and labor market demands and the incorporation of new educational technologies. Based on this analysis, we created a group of subjects common to all health courses taught at the institution, i.e., an identity that characterizes the profile of the professional graduating from the Health Sciences Center at Sagrado Coração University was developed. The results were the training of a professional competent to act as a manager and multiplier of the NHS principles, the improvement of the training process of the professionals in service, and the promotion of multidisciplinary work. For users, meanwhile, the results translate in improvements in the quality of the services received. Keywords Curriculum; health; primary care. 


\section{Considerações iniciais}

As três diretrizes ordenadoras do Sistema Único de Saúde (SUS) são autênticos desafios: a descentralização da gestão com comando único por esfera de governo, a participação popular e o atendimento integral, com prioridade para as atividades preventivas, sem prejuízo dos serviços assistenciais. Descentralização, universalidade e integralidade da atenção constituem uma tríade de princípios que expressam em grande medida o processo de consolidação de conquistas do direito à saúde como uma questão de cidadania (Feuerwerker e Almeida, 2003).

As diretrizes curriculares para os cursos da área da saúde, aprovadas entre 2001 e 2002, indicam que a formação do profissional deve contemplar o sistema de saúde vigente no país, o trabalho em equipe e a atenção integral (Ceccim e Feuerwerker, 2004).

A formação em saúde coletiva deve desenvolver competências para o cuidado individual e coletivo, resultando na qualificação de profissionais críticos e comprometidos com a melhoria da saúde e com sua performance na equipe (De Carvalho e Ceccim, 2006).

A reestruturação curricular com foco na avaliação da aprendizagem possibilitará a formação de profissionais preparados para atuarem com as reais necessidades do indivíduo e da coletividade em que atua (Higa et al., 2013).

Assim, as novas diretrizes constituem o eixo central do processo de mudança no ensino superior, mas novos desafios surgem, como a formação e educação continuada dos docentes, a introdução de inovações pedagógicas no processo ensino-aprendizagem e a inserção dos docentes e estudantes nas práticas dos serviços de saúde (Teixeira, 2003).

Da necessidade de fortalecer a integração das práticas de ensino com as instâncias do SUS fez-se a reorientação das matrizes curriculares dos cursos da área da saúde. Para efetividade de tal reorientação, a consolidação da parceria entre a instituição de ensino superior (IES) e a secretaria municipal de saúde foi essencial em todos os cenários de atuação profissional, o que resultou na elaboração em conjunto do Programa Nacional de Reorientação da Formação Profissional em Saúde II (Pró-Saúde II), com aprovação dos ministérios da Saúde e da Educação (Brasil, 2007).

A integração entre ensino e serviço proporciona melhor capacitação do docente, do estudante e do profissional do serviço de saúde municipal, consequentemente garante ações e serviços de qualidade à população, por meio de reorientação da atenção básica e do modelo de atenção à saúde vigente no sistema nacional. Portanto, o objetivo deste artigo é descrever a realização da reorientação da formação dos profissionais da saúde na Universidade do Sagrado Coração (USC), proporcionando a integração com a rede básica. 


\section{A Universidade do Sagrado Coração}

A USC, localizada em Bauru, recebeu autorização para funcionar no ano de 1953. A cidade de Bauru está situada na região sudeste do estado de São Paulo, numa área de $702 \mathrm{~km} 2$, a $324 \mathrm{~km}$ da capital, com altitude média de 526 m, numa região cuja vegetação nativa é o cerrado. O Censo de 2010 afirma que a população de Bauru era de 316.064 habitantes (Instituto Brasileiro de Geografia e Estatística, 2010).

Na década de 1970 foram instalados os cursos de Ciência (Habilitação em Biologia) e Enfermagem; na de 1980, os de Farmácia, Nutrição e Fonoaudiologia; e na de 1990, os de Fisioterapia, Odontologia e Terapia Ocupacional (Universidade Sagrado Coração, 1998).

O campus universitário em 2013 contava com aproximadamente seis mil estudantes distribuídos em 42 cursos de graduação, dentre os quais nove na área da saúde. Além da graduação, há diversos cursos lato sensu e programas stricto sensu. O corpo docente é composto por 285 professores qualificados e com apoio de uma equipe técnica de sustentação com cerca de 234 colaboradores.

A USC, como instituição católica, busca construir um trabalho educativo capaz de proporcionar formação sólida no aspecto cultural, ético e profissional, privilegiando o desenvolvimento pleno da pessoa, seu preparo para o exercício da cidadania, qualificação para o trabalho e domínio de competências profissionais.

O Centro de Ciências da Saúde (CCS) é uma unidade que congrega cursos e gerencia o exercício simultâneo do ensino, da pesquisa e da extensão, nas áreas de conhecimento cobertas pelas ciências da saúde.

Os esforços do CCS estão centrados na incorporação de uma perspectiva interdisciplinar, a qual abrange o indivíduo, os grupos, as comunidades e as esferas institucionais, e busca tanto desenvolver nos estudantes competências para atuarem no sistema de saúde com intervenções não apenas curativas como vislumbra um cenário no qual a promoção da saúde e a prevenção passam a ser o eixo principal na formação profissional.

Um estudo sobre alteração de matriz curricular pautado nas recomendações das diretrizes do SUS, buscando equilibrar excelência, técnica e relevância social, desenvolvido por De Paula Ferreira et al. (2012), evidenciou que ocorreu a integração do conhecimento entre as disciplinas, fortalecendo os procedimentos preventivos, uma experiência significativa na formação profissional.

Atualmente, o desafio é sustentar um dos pilares da formação inovadora que é o universo da saúde coletiva humanizada. 


\section{A Secretaria Municipal da Saúde}

Em 2013, a Secretaria Municipal de Saúde (SMS) de Bauru congregava 25 Unidades Básicas de Saúde (UBS), oito Serviços de Atendimento de Urgência e Emergência (um vinculado à UBS) e oito Unidades e Serviços Especializados.

Desenvolveu 13 programas, a saber: controle da hipertensão arterial e do diabetes mellitus; saúde do trabalhador; saúde mental; atenção às pessoas portadoras de transtornos mentais; atenção ao idoso; saúde bucal, incluindo a atenção odontológica básica e a prevenção de câncer bucal; orientação e prevenção do câncer; assistência farmacêutica; controle de zoonoses; controle das doenças sexualmente transmissíveis e Aids; controle da hanseníase, controle da tuberculose; saúde da criança, incluindo: defesa da vida do lactente, puericultura, saúde bucal do bebê, saúde ocular do escolar, atenção à nutrição infantil e suplementação de nutrientes especiais; saúde da mulher, incluindo atenção ao pré-natal, saúde bucal da gestante, prevenção de câncer de colo de útero e de mamas, planejamento familiar e saúde da família.

\section{Reestruturação das matrizes curriculares}

Para esse trabalho, optamos pela abordagem qualitativa documental, com análise das Diretrizes Curriculares Nacionais (DCN) para os Cursos da Área da Saúde (Brasil, 2002), dos projetos pedagógicos dos Cursos da USC e das Diretrizes Operacionais do Pacto pela Saúde do SUS (Brasil, 2006).

Nesse sentido, as DCN têm papel norteador nas alterações curriculares e consequentemente na mudança no cenário do ensino de graduação dos futuros profissionais da saúde, à medida que a orientam a construção do perfil do egresso na área da saúde (Pereira e Lages, 2013).

No período de 2006 a 2008, o processo de estudos e reestruturação das matrizes curriculares da USC ocorreu por meio de discussões, em reuniões de colegiados, com oficinas de docentes e discentes que confrontavam as matrizes curriculares até então vigentes com a proposta que implicava a necessidade da aproximação de competências e habilidades, e consequente reorientação na formação dos profissionais, com ampliação do conceito de saúde e integração entre os vários cursos de saúde da instituição.

Os cursos de graduação do CCS apresentavam até o ano de 2007 disciplinas que contemplavam as atuações voltadas à intervenção nos diferentes níveis de atenção à saúde. Estas intervenções caracterizavam-se por ações isoladas, não proporcionando aos estudantes a visão integral do processo de assistência à saúde-doença e da interdisciplinaridade. 
A visão biologicista da doença e o enfoque no adestramento técnico da assistência clínico-reabilitadora, agregados a questões didáticas e estruturais, não aproximavam a formação acadêmica das reais necessidades da maioria da sociedade brasileira, o que restringia a perspectiva da prática e, muitas vezes, frustrava os egressos que se deparavam com a realidade ao saírem das instituições de ensino (Feuerwerker, 2000).

Para efetivação do processo de reestruturação das matrizes curriculares, a equipe diretiva da USC e os coordenadores dos cursos participaram da capacitação "Construção de matrizes curriculares baseadas em competências", possibilitando reflexões sobre as competências estabelecidas pelas diretrizes e pelo SUS. Após este processo, os coordenadores, apoiados pela equipe diretiva, constituíram-se em multiplicadores dos conceitos e discutiram as propostas com o corpo docente, o que proporcionou a criação de novas matrizes curriculares articuladas e integradas.

O conceito de competência existente nas DCN ampliou a responsabilidade da USC como IES; exigiu a inclusão de novos conteúdos, de novas formas de organização do trabalho, da incorporação dos conhecimentos que são adquiridos na prática, de metodologia que desenvolvam a capacidade de como resolver novos problemas, comunicar ideias, tomar decisões e trabalhar em equipe (Meyer e Kruse, 2003).

A defesa da ideia de competência profissional como capacidade de mobilizar recursos e conhecimentos implica, necessariamente, um modelo de formação que garanta o desenvolvimento progressivo de habilidades, que se espera dos profissionais da saúde (Brasil, 2001; Batista e Gonçalves, 2011).

Para o projeto de remodelação das estruturas curriculares dos cursos da área de saúde na USC, a busca de formação por competências inverteu a lógica que tradicionalmente conduzia a organização curricular. Ao invés de partir de uma listagem de disciplinas obrigatórias e respectivas cargas horárias, a nova proposta exigiu como referência inicial o conjunto de competências que se esperava do egresso e o respectivo conjunto de competências próprias para cada profissão.

Considerando-se, ainda, que a graduação dura somente alguns anos, enquanto a atividade profissional pode permanecer por décadas e que os conhecimentos e competências se transformam velozmente, torna-se essencial pensar em uma metodologia para uma prática de educação libertadora, na formação de um profissional ativo e apto a aprender a aprender. As metodologias ativas utilizam a problematização como estratégia de ensino-aprendizagem, com o objetivo de alcançar e motivar o discente, que, diante do problema, se detém, examina, reflete, relaciona a sua história e passa a ressignificar suas descobertas (Mitre et al., 2008; Lazzarin, Nakama, Cordoni Junior, 2010). 
Colomé e Oliveira (2012) afirmam que a responsabilidade é das instituições educacionais com a formação de profissionais da saúde conscientes das necessidades do SUS. Assim, verificamos a necessidade de incrementar a integração das práticas de ensino com as instâncias do SUS, pois as interações proporcionadas por alguns segmentos dos vários cursos não causavam a repercussão esperada dos docentes e discentes, sendo imprescindível consolidar essas interações e efetivá-las em processos durante todo o curso.

Dias, Lima e Teixeira (2013) também constataram a importância de maior integração e participação da sociedade loco-regional. Outro aspecto relevante na reestruturação das matrizes se relaciona com práticas de ensino no sentido de se contemplar o aprender a aprender, o aprender a fazer, o aprender a viver junto e o aprender a ser (Morin, 2000).

Esta análise da equipe de trabalho sinalizou a necessidade de produzir conhecimentos sobre o quadro epidemiológico da população e das políticas de saúde que regem o sistema de saúde do Brasil, a fim de identificar quais são os conhecimentos relevantes para a melhoria do quadro de saúde da população. A partir desta análise, criou-se um grupo de disciplinas comum a todos os cursos de saúde da instituição para o desenvolvimento de uma identidade, que caracteriza o perfil do profissional dos cursos do CCS da USC.

O objetivo principal da criação desse grupo de disciplinas foi o de garantir ao discente o desenvolvimento de competências e habilidades para um perfil profissional generalista capaz de atuar de forma integral e interdisciplinar no processo de assistência à saúde-doença.

Entre essas disciplinas destacam-se:

a) Politicas e Gestão da Saúde, cujo objetivo é capacitar o estudante a identificar políticas e tipos de Estado; identificar e analisar as políticas públicas de saúde historicamente construídas no Brasil; identificar as políticas públicas de saúde na atualidade (reforma sanitária, SUS e modelos de saúde complementares); conhecer e participar do Conselho Municipal de Saúde; conhecer as metas estabelecidas no Pacto de Saúde; caracterizar os modelos de gestão em saúde e compreender e utilizar as tecnologias e sistemas de informação em saúde;

b) Educação em Saúde, com objetivo de capacitar o estudante a conceituar educação em saúde; relacionar a função educativa com a aplicação de princípios didáticos na prática profissional; utilizar adequadamente procedimentos didáticos e tecnologia de ensino; desenvolver atividade educativa em diferentes cenários, com responsabilidade e ética, de modo que estimulem a comunidade a adotar e manter padrões de vida sadios, usar de forma judiciosa e cuidadosa os serviços de saúde colocados à sua disposição, e tomar sua próprias decisões, tanto individual como coletivamente, visando a melhorar suas condições de saúde e as condições do meio ambiente. 
Com esta proposta, os egressos da USC poderão, como futuros membros dos recursos humanos da saúde no país, atuar adequadamente, no sentido de garantir ações e serviços de qualidade à população, por meio de reorientação da atenção básica e do modelo de atenção à saúde no sistema nacional vigente.

Ao considerar que as preocupações referentes aos recursos humanos em saúde vêm de longa data, a USC buscou reorganizar as estruturas curriculares dos cursos do CCS no sentido de formar profissionais capacitados e habilitados para atuação junto ao sistema de saúde vigente no país.

O SUS constitui efetivamente um campo de trabalho para o profissional de saúde, e a população deve receber um padrão de serviços compatível com a melhoria da qualidade de vida, com maior resolubilidade da atenção e garantia de acesso aos demais níveis do sistema (Brasil, 2004).

Ponderando tais aspectos, a reorganização proposta inclui a adequação do ensino às demandas sociais, às do mercado de trabalho e à incorporação de novas tecnologias educacionais ao currículo, visando a autonomia dos estudantes e a qualidade do trabalho docente. Porém, compartilha-se do posicionamento de Pereira e Lages (2013) no que diz respeito à utilização da Teoria Crítica na composição de matrizes curriculares para saúde, a qual não responde a todos os desafios, contudo, contribui na transformação do cenário da saúde. Também se compartilha com Ferreira et al. (2014) na proposta de uma ação pedagógica com comunicação bilateral, geradora de cidadania.

Com foco na mudança, além da reestruturação das matrizes curriculares, a USC iniciou o Projeto da Coordenadoria Pedagógica, o qual representa um compromisso com o futuro acadêmico, que inovou a metodologia de ensino-aprendizagem, visando a ter um corpo docente facilitador deste processo.

A Coordenadoria Pedagógica se institui como um espaço de atendimento, orientação e desenvolvimento de capacitação para os docentes, com o objetivo de contribuir para a operacionalização do Modelo Pedagógico Syllabus instituído como metodologia para os alunos iniciantes do ano letivo de 2008 (Santos et al., 2011).

A escolha do Modelo Pedagógico Syllabus foi decorrente da assessoria administrativa e acadêmica oferecida pela Universidade Santo Tomás do Chile que, desde 1998, fundamenta suas práticas educativas com o objetivo de alcançar excelência universitária. A terminologia Syllabus é de origem inglesa e, dentre outros significados, tem o sentido de detalhar, enumerar, especificar os componentes de um plano de atividades (Santos e Nakashima, 2008).

Esse modelo pedagógico se fundamenta no enfoque construtivista de aprendizagem, no processo ativo de construção a partir do conhecimento do estudante e do planejamento sistemático pelo professor das atividades do Plano de Ensino da disciplina, no contexto individual e coletivo, a fim de tornar a sala de aula uma ocasião de participação, tanto do estudante como do professor (Schiefelbein e Zúñiga, 2002; Vatter e Novoa, 2005). 
O envolvimento dos estudantes ocorre por meio da compreensão das orientações de atividades prévias à aula presencial, disponibilizadas pelo docente em ambiente virtual, e avaliado com a sua participação no Quiz, no início de cada aula. O Modelo Pedagógico Syllabus propõe uma metodologia de interação entre o professor e o estudante, para facilitar a aprendizagem e construção do conhecimento em sala de aula (Zuccari et al., 2010).

\section{Próximos passos}

Para estender as mudanças além do âmbito universitário e efetivá-las no sistema e nos cursos de saúde, o corpo diretivo da USC e a SMS do município de Bauru propõem firmar a relação existente entre saúde e educação por meio da adequação dos profissionais às necessidades sociais da população.

A adequação inclui a participação do corpo docente e discente da USC e dos profissionais da saúde da rede municipal, que em conjunto farão um diagnóstico das necessidades dos usuários nos diferentes aspectos. Tal diagnóstico basear-se-á em conceitos teóricos e nos registros dos programas desenvolvidos pela rede, além dos dados epidemiológicos do município. As informações, analisadas criteriosamente por profissionais das diferentes áreas da saúde, apoiados por conhecimentos tecnológicos, bases científicas e instrumentais, proporcionarão o estabelecimento de estratégias específicas, interligando a teoria à prática.

As estratégias desenvolvidas terão como objetivo principal a formação do profissional voltada ao processo saúde-doença e a capacitação dos profissionais da rede municipal para intervirem adequadamente na realidade local, promovendo a saúde e prevenindo ou reabilitando a doença (Brasil, 2007).

Os discentes da USC terão a oportunidade de atuar diretamente com os profissionais de saúde da rede. Tal atuação contempla um processo dinâmico entre docentes, profissionais da saúde e estudantes, no qual todos os envolvidos participarão ativamente. Em determinados momentos, os estudantes, apoiados pelo corpo docente, desenvolverão estratégias de capacitação para os profissionais, elaborarão materiais de informação e divulgação para os profissionais da rede e os usuários. Em outras situações, esses profissionais e usuários contemplarão os estudantes com seus relatos e experiências.

Desta capacitação interativa surgirão propostas metodológicas, que poderão ser aplicadas diretamente ao usuário, o que proporcionará, desta forma, integração entre instituição de ensino, serviço de saúde e comunidade assistida. 


\section{Considerações finais}

Com tal proposta, espera-se formar o profissional da saúde com competência para atuar no SUS como gestor e multiplicador dos princípios da promoção da saúde e prevenção e reabilitação das doenças.

A distribuição das disciplinas, o caráter teórico-prático, o contato precoce do estudante com a realidade de saúde do país, a aplicação direta voltada às políticas de saúde local, e a formação articulada, integrada e interdisciplinar conduzirão à compreensão dos aspectos biológicos, ecológicos, sociais e comportamentais, em níveis individual e coletivo, do processo saúde-doença. Contribuirão ainda para a formação de profissionais de saúde, aptos a atuar, garan-tindo ações e serviços de saúde de qualidade à população.

A inserção de disciplinas comuns a todos os cursos do CCS, com o foco em Políticas e Gestão de Saúde e Educação para Saúde, desenvolverá no profissional em formação a capacidade gerencial e a habilidade para criar e aplicar estratégias educativas.

Com a integração ensino-serviço, espera-se, além de contribuir e fortalecer a formação capacitada do estudante para atuação nos diferentes cenários de atenção à saúde, incrementar o processo de capacitação do profissional em serviço, assim como promover o trabalho multiprofissional em todos os níveis do sistema.

Para os usuários, o resultado deve reverter-se em melhoria da qualidade dos serviços recebidos, além de facilitar a busca de informações em fontes seguras e de fácil acesso. Tais esclarecimentos poderão facilitar para o usuário a compreensão e participação ativa no processo de promoção da saúde, prevenção e reabilitação da doença, oferecendo também aos servidores, profissionais em formação e docentes dados que subsidiarão o desenvolvimento de novas e constantes estratégias para atender a real necessidade da comunidade assistida.

\section{Colaboradores}

Todos os autores participaram igualmente de todas as fases de elaboração do artigo, desde a concepção, análise dos dados até a escrita do texto propriamente dito. Não há conflitos de interesse. 
Resumen La integración entre enseñanza y servicio ofrece una mejor capacitación del docente, del estudiante y del profesional del servicio de salud, y así asegura acciones y servicios de calidad a la población. Este estudio tuvo como objetivo reorientar la formación de los profesionales de los cursos del Centro de Ciencias de la Salud de la Universidad Sagrado Corazón, Bauru, São Paulo, Brasil, en consonancia con el Programa Nacional de Reorientación de la Formación Profesional en Salud II. En estos cursos, se reorganizaron las matrices curriculares, incluyendo la adecuación de la enseñanza a las demandas sociales, del mercado de trabajo y la incorporación de nuevas tecnologías educativas. A partir de este análisis, se creó un grupo de disciplinas común a todos los cursos de salud de la institución, es decir, se desarrolló una identidad que caracteriza el perfil del profesional de los cursos del Centro de Ciencias de la Salud de la Universidad Sagrado Corazón. Los resultados fueron formación del profesional con competencia para actuar como gestor y multiplicador de los principios del SUS, el incremento en el proceso de capacitación del profesional en servicio y la promoción del trabajo multiprofesional. Para los usuarios, los resultados se revierten en mejora de la calidad de los servicios recibidos.

Palabras clave matriz curricular; salud; atención básica.

\section{Notas}

1 Universidade Sagrado Coração, Centro de Ciências da Saúde, Bauru, São Paulo, Brasil.

$<$ leila.vieira@usc.br>

Correspondência: Rua Irmã Arminda, 10-50, Jardim Brasil, CEP 17030-620, Bauru, São Paulo, Brasil.

2 Fundação Estatal Regional de Saúde, Bauru, São Paulo, Brasil.

$<$ fundacao.saude@bauru.sp.gov.br>

3 Universidade Sagrado Coração, Centro de Ciências da Saúde, Bauru, São Paulo, Brasil. <esimionato@ufsc.br>

4 Universidade Sagrado Coração, Centro de Ciências da Saúde, Bauru, São Paulo, Brasil. <einoue@usc.br>

5 Universidade Sagrado Coração, Centro de Ciências da Saúde, Bauru, São Paulo, Brasil. $<$ mheubel@usc.br>

6 Universidade Sagrado Coração, Centro de Ciências da Saúde, Bauru, São Paulo, Brasil. <madeconti@yahoo.com.br>

7 Universidade do Sagrado Coração, Coordenadoria Geral de Extensão, Bauru, São Paulo, Brasil.

<sandra.saes@usc.br> 


\section{Referências}

BATISTA, Karina B. C.; GONÇALVES, Otília S. J. Formação dos profissionais de saúde para o SUS: significado e cuidado. Saúde e Sociedade, São Paulo, v. 20, n. 4, p. 884-899, 2011.

BRASIL. Ministério da Educação. Secretaria de Educação Fundamental. Programa de Formação de Professores Alfabetizadores. Guia de Orientações Metodológicas Gerais. Janeiro 2001. Disponível em: <http://portal. mec.gov.br/seb/arquivos/pdf/guia_orient. pdf>. Acesso em: 22 ago. 2013.

BRASIL. Ministério da Saúde. Ministério da Educação. Programa de Reorientação da Formação Profissional em Saúde - Pró-Saúde: objetivos, implementação e desenvolvimento potencial. Brasília, DF: MS; MEC, 2007.

BRASIL. Ministério da Saúde. Política nacional de humanização: marco conceitual e diretrizes políticas. Brasília: Ministério da Saúde, 2004

BRASIL. Ministério da Saúde. Secretaria Executiva. Diretrizes operacionais dos Pactos pela Vida, em Defesa do SUS e de Gestão. Brasília: 76 p. (Série A. Normas e Manuais Técnicos). 2006. Disponível em: <http:// portal.saude.gov.br/portal/arquivos/pdf/ volume_1_completo.pdf $>$. Acesso em: 16 ago. 2013.

BRASIL. Portaria interministerial n. 610 de 26 de março de 2002. Institui o Programa Nacional de Incentivo às Mudanças Curriculares para as Escolas Médicas. Diário Oficial da República Federativa do Brasil, Brasília, DF, 26 mar. 2002.

CECCIM, Ricardo B.; FEUERWERKER, Laura C. M. Mudança na graduação das profissões de saúde sob o eixo da integralidade. Cadernos de Saúde Pública, Rio de Janeiro, v. 20, n. 5 , out. 2004 .

COLOMÉ, Juliana S.; OLIVEIRA, Dora L. L. C. Educação em saúde: por quem e para quem? A visão de estudantes de graduação em Enfermagem. Texto \& Contexto Enfermagem, Florianópolis, v. 21, n. 1, p. 177-184, 2012.

DE CARVALHO, Yara M.; CECCIM, Ricardo B. Formação e educação em saúde: aprendizados com a saúde coletiva. In: CAMPOS, Gastão W. S. (org.). Tratado da saúde coletiva. Rio de Janeiro: Fiocruz, 2006. p. 137-170.

DE PAULA FERREIRA, Naiara et al. Clínica integrada e mudança curricular: desempenho clínico na perspectiva da integralidade. Pesquisa Brasileira em Odontopediatria e Clínica Integrada, Goiânia, v. 12, n. 1, p. 33-39, 2012.

DIAS, Henrique S.; LIMA, Luciana D.; TEIXEIRA, M. A trajetória política de reorientação da formação profissional em saúde no SUS. Ciência \& Saúde Coletiva, Rio de Janeiro, v. 18, n. 6, p. 1.613-1.624, jul. 2013.

FERREIRA, Viviane F. et al. Educação em saúde e cidadania: revisão integrada. Trabalho, Educação e Saúde, Rio de Janeiro, v. 12, n. 2, p. 363-378, maio-ago. 2014.

FEUERWERKER, Laura C. M. Além do discurso da mudança na educação médica: processos e resultados. Rio de Janeiro: Hucitec, 2002.

FEUERWERKER, Laura C. M.; ALMEIDA, Marcio. Diretrizes curriculares e projetos pedagógicos: é tempo de ação! Revista Brasileira de Enfermagem, Brasília, v. 56, n. 4, p. 351-352, jul.-ago. 2003.

HIGA, Elza F. R. et al. Indicadores de avaliação em gestão e saúde coletiva na formação médica. Revista Brasileira de Educação Médica, Rio de Janeiro, v. 37, n. 1, mar. 2013. Disponível em: <www.scielo.br/scielo.php?script= sci_arttext\&pid=S0100-55022013000100008\& $\operatorname{lng}=$ pt\&nrm=iso $>$. Acesso em: 1 set. 2013.

INSTITUTO BRASILEIRO DE GEOGRAFIA E ESTATÍSTICA (IBGE). Sinopse do censo demográfico 2010. Disponível em: <www.censo2010. 
ibge.gov.br/sinopse/index.php?uf $=35 \&$ dados $=0>$. Acesso em 01 set. 2013.

LAZZARIN, Helen C.; NAKAMA, Luiza; CORDONI-JÚNIOR, Luiz. Percepção de professores de odontologia no processo de ensino aprendizagem. Ciência \& Saúde Coletiva, Rio de Janeiro, v. 15, supl. 1, p. 1.801-1.910, 2010.

MEYER, Dagmar E.; KRUSE, Maria H. L. Acerca de diretrizes curriculares e projetos pedagógicos: um início de reflexão. Revista Brasileira de Enfermagem, Brasília, v. 56, n. 4, p.335-339, ago. 2003.

MITRE, Sandra M. et al. Metodologias ativas de ensino-aprendizagem na formação profissional em saúde: debates atuais. Ciência \& Saúde Coletiva, Rio de Janeiro, v. 13, supl. 2, p. 2.133-2.144, 2008. Disponível em: <www. scielo.br/pdf/csc/v13s2/v13s2a18.pdf $>$. Acesso em: 23 ago. 2013.

MORIN, Edgar. Os sete saberes necessários à educação do futuro. São Paulo: Cortez, 2000.

PEREIRA, Ingrid D. F.; LAGES, Itamar. Diretrizes curriculares para a formação de profissionais de saúde: competências ou práxis? Trabalho, Educação e Saúde, Rio de Janeiro, v. 11, n. 2, p. 319-338, maio-ago. 2013. Disponível em: <www.revista.epsjv.fiocruz.br/ upload/revistas/r486.pdf $>$. Acesso em: 24 ago. 2013.

SANTOS, Marisa A. P. et al. Avaliar projeto metodológico: isto é possível?. Avaliação (Campinas), Sorocaba, v. 16, n. 2, jul. 2011. Disponível em: <www.scielo.br/scielo.php?script= sci_arttext\&pid=S1414-40772011000200011\& lng $=$ en \&nrm=iso > . Acesso em: 22 fev. 2013.
SANTOS, Marisa A. P.; NAKASHIMA, Rosária H. R. Model didático-pedagógico Syllabus: a prática metodológica no ensino superior associada aos programas de estudos, planejamento e avaliação. In: CONGRESSO NACIONAL DE EDUCAÇÃO DA PUCPR, 8. - EDUCERE, 10., 2008, Curitiba. Anais..., Curitiba: PUCPR, 2008. Disponível em: <www.pucpr.br/eventos/ educere/educere2008/anais/pdf/503_949.pdf > . Acesso em: 28 out. 2013.

SCHIEFELBEIN, Ernesto; ZÚÑIGA, Ricardo. El Syllabus: viviendo un aprendizaje autónomo. Bogotá: Universidad Santo Tomás, 2002.

TEIXEIRA, Carmen F. Ensino da saúde coletiva na graduação. Boletim da Abem, Rio de Janeiro, v. 31, n.3, p. 20-22, maio-jun. 2003.

UNIVERSIDADE DO SAGRADO CORAÇÃO. Identidade e missão. Bauru: USC, 1998.

VATTER, Jaime; NOVOA, Daniela. Metodología de enseñanza-aprendizaje Syllabus. In: ENCUENTRO DE RECTORES UNIVERSIA, 2. Santiago do Chile. Anais..., Santiago: Universia, 2005. p. 61-67.

ZUCCARI, Patrícia et al. Planejamento e avaliação: pilares da gestão do modelo pedagógico Syllabus. Política e Gestão Educacional, Araraquara, v. 9, p. 1-13, 2010. Disponível em: $<$ www.fclar.unesp.br/Home/Departamentos/ CienciasdaEducacao/RevistaEletronica/edi9_ artigo7.pdf > . Acesso em: 10 nov. 2012.

Recebido em 26/02/2013

Aprovado em 05/03/2015 